PSICOLOGÍA

IBEROAMERICANA
Psicología Iberoamericana ISSN: 1405-0943

revista.psicologia@ibero.mx

Universidad Iberoamericana, Ciudad de México México

\title{
La amenaza detrás de la belleza: Un análisis psicológico en modelos de moda mexicanos
}

Rodríguez Peña, Gilberto Javier; Moreno Almazán, Omar

La amenaza detrás de la belleza: Un análisis psicológico en modelos de moda mexicanos

Psicología Iberoamericana, vol. 27, núm. 1, 2019

Universidad Iberoamericana, Ciudad de México, México

Disponible en: http://www.redalyc.org/articulo.oa?id=133960951008 


\title{
La amenaza detrás de la belleza: Un análisis psicológico en modelos de moda mexicanos
}

\author{
The menace behind beauty: A psychological analysis of \\ Mexican fashion models \\ Gilberto Javier Rodríguez Peña gilbertopen1993@gmail.com \\ Universidad Tecnológica de México-Atizapán, México \\ http://orcid.org/0000-0002-3662-9295 \\ Omar Moreno Almazán \\ Universidad Nacional Autónoma de México, México \\ http://orcid.org/0000-0001-9562-0234
}

Psicología Iberoamericana, vol. 27, núm. 1,2019

Universidad Iberoamericana, Ciudad de México, México

Recepción: 12 Junio 2019

Aprobación: 19 Septiembre 2019

Redalyc: http://www.redalyc.org/ articulo.oa?id=133960951008
Resumen: El presente trabajo tiene por objetivo conocer la influencia que tienen los medios de comunicación habituales y los hábitos alimenticios sobre la imagen corporal y autoestima. La metodología se centró en la aplicación de diversas pruebas psicométricas sobre una muestra representativa de 100 personas practicantes de actividades relacionadas con el modelaje, con edades de 18 a 27 años. Se realizaron algunos análisis basados principalmente en el sexo de los participantes, mostrando algunos efectos negativos para ellos. Por otro lado, las variables edad y tiempo en la labor de modelaje no fueron importantes. Como conclusión principal, se encontró que los hábitos alimenticios y medios de comunicación poseen relación y que pueden mermar la autoestima de los modelos y al mismo tiempo el deterioro de la imagen corporal. De esta forma, el presente estudio muestra datos que pueden ser útiles para investigaciones futuras e intervenciones próximas en muestras mayores.

Palabras clave: imagen corporal, autoestima, alimentación, comunicación.

Abstract: The aim of this paper is to understand the influence of the media and eating habits on body image and self-esteem. The methodology focused on the application of various psychometric tests on a representative sample of 100 people involved in activities related to modeling, aged 18 to 27 years. Some analyses were carried out based mainly on the sex of the participants, showing some negative effects for them. On the other hand, this study does not show age or the time spent working in the field of modelling as important variables, leading to the conclusion that eating habits and media are related and may lower self-esteem and lead to the deterioration of one's body image. This study is useful for future research and upcoming interventions in larger samples.

Keywords: body image, self-concept, nutrition, communication.

\section{Introducción}

Los modelos estéticos emergieron por primera vez desde los tiempos finales de la primera Guerra Mundial, tardaron en difundirse en todo el planeta al inicio de la segunda Guerra Mundial con un intervalo de veinticinco años; sin embargo, a partir de 1960 fue cuando la imagen corporal se volvió una obsesión (Bazán \& Miño, 2015). En éste sentido, se maneja el término de la imagen corporal refiriéndose a los sentimientos de una persona sobre la estética y el atractivo sexual del propio cuerpo (Padrón-Salas et al., 2015), tales como la 
figura corporal, los gestos, la postura, las maneras y los estilos de vestir (Gil Madrona, 2013). Por otra parte, el término autoestima se define como el aprecio de una persona sobre sí misma (Padrón-Salas et al., 2015), y que en su condición negativa o escasa, puede entenderse como una estructura de la imagen corporal de una persona a partir de las siguientes dimensiones: nivel psicofisiológico, que son respuestas de activación del Sistema Nervioso Autónomo (sudor, temblor, dificultad de respiración, etcétera.); nivel conductual, que incluye conductas de evitación, camuflaje, rituales de comprobación, realización de dieta, ejercicio físico, etcétera; nivel cognitivo-afectivo, como preocupación intensa, creencias irracionales, distorsiones perceptivas, sobreestimación y subestimación, tristeza, vergüenza, etcétera; nivel perceptual, que es la forma como se percibe el tamaño de diferentes segmentos corporales (Raich, 2004). Se piensa que uno de los grupos más afectados es el de los modelos de moda, considerando que en ésta industria hay presión sobre el mantenimiento de la figura delgada. Es tal la presión, que algunas agencias de modelaje influyen para que las trabajadoras pierdan peso, aun sabiendo que las modelos de moda tiene cada vez más conocimiento acerca de los derechos que las protegen. Con el tiempo incluso puede llegar a desarrollarse un ambiente tóxico y peligroso para la percepción de la imagen corporal (Chacón Araya, 2017). La idea de la figura delgada crea una expectativa irreal entre las mujeres, quienes opinan que la extrema delgadez les brinda mayor éxito y atención como modelos, lo que puede resultar en una imagen corporal negativa, convirtiéndose en un peligroso grupo para enfermedades afectivas (Swami \& Szmigielska, 2013).

En ocasiones se llegó a denominar el cuerpo de una modelo como algo "infeccioso", ya que muchas mujeres dedicadas a ésta profesión influyen en otras mujeres o modelos más jóvenes de manera negativa y pueden relacionarse con la anorexia. Se afirma que la delgadez extrema y palidez son signos de advertencia para determinar la delgada línea entre la belleza y la salud (Díaz Soloaga, Quintas Froufe, \& Muñiz, 2010). En un estudio histórico, Garner, Garfinkel, Schwartz y Thompson (1980) destacan que desde 1960 a 1979 las modelos de las páginas centrales de Playboy y las concursantes de Miss America se fueron haciendo cada vez más delgadas. Para dicho estudio revisaron el peso y la altura de las modelos y encontraron una disminución de peso de $3.4 \mathrm{~kg}$ y también de las dimensiones de pechos y caderas. Por otra parte, en el caso de los varones, el modelo dominante es excesivamente musculoso (Soto Ruiz et al., 2015). Leit, Gray y Pope (2001) señalan que los modelos masculinos de la revista Play girl, de 1973 a 1997, se fueron haciendo cada vez más musculosos.

\section{Autoestima e Imagen Corporal}

La relación entre éstas dos (imagen corporal-autoestima) se basa en que la autoestima estará determinada por el agrado que la persona tiene respecto a su imagen. Si está insatisfecho respecto a su imagen, la autoestima se verá disminuida, abriendo la puerta incluso al trastorno dismorfico 
corporal o depresión grave (Esteves, 2012). La autoestima e imagen corporal impulsan a la persona a luchar por dar una imagen hacia la sociedad, lo que le permite recibir buenas críticas, aceptación social, y desde luego existe una mejora en la autoestima de la persona una vez que alcanzó el canon de belleza deseado (Prieto Vela \& Muñoz-Najar Pacheco, 2015). Por otro lado, se hace la mención que estar delgado ya es una presión de la sociedad, incluso puede llegar a ser señal de exclusión en los grupos de pares de una persona (Nieves, 2013). Esto hace que la imagen de corporalidad y autoestima de una persona esté condicionada a las opiniones y miradas de los otros (Saüch \& Castañer, 2013). San Mauro et al. (2014) reveló en su estudio una muestra ajena al modelaje profesional en que al $58 \%$ de las mujeres les gusta que admiren su apariencia; situación inversa en el grupo de los hombres entre quienes solo el $44 \%$ gusta de esa admiración. Además, un $20 \%$ de los hombres y un 33\% de las mujeres reconocieron que se comparan físicamente con otras personas con frecuencia y, conjuntando otro dato, tanto hombres como mujeres (el $45 \%$ de ellos y el $66 \%$ de ellas) se preocupan por su aspecto físico y comprueban su imagen en el espejo con regularidad (San Mauro et al., 2014). Meyer, Enström, Harstveit, Bowles y Beevers (2007), demostraron en su estudio con 56 modelos de moda profesionales que poseen una necesidad mayor de satisfacción, imagen de corporalidad, admiración y aceptación; al mismo tiempo, una correlación de éstas con un estado anímico bajo si no se alcanzan en comparación con las personas que no ejercen el modelaje.

\section{Medios de Comunicación}

El perfil del canon de belleza y la presión por dar una mejor impresión a la sociedad hacen que la atención de la persona recaiga en los medios de comunicación, donde observan cómo productos para bajar de peso son promocionados por figuras públicas reconocidas que logran influir en ese deseo; si no se logra el cuerpo delgado, se considera un fracaso para la persona. Esto se da aún más en la cultura de los modelos de moda, ya que es un universo en donde la delgadez promovida es un requisito (Bogár \& Túry, 2018). Los medios de comunicación tales como revistas, comerciales televisivos, etcétera. tienen una potencial influencia psicológica en las personas. Los estereotipos de belleza de extrema delgadez imperan en las mujeres (Bazán \& Miño, 2015), mientras que ahora en determinados anuncios abunda la imagen de modelos masculinos con mayor desarrollo y definición muscular, los cuales se han ligado con valores de éxito y estatus. Dichas características se han tratado de interiorizar tanto al resto de la sociedad, como a las futuras generaciones de modelaje (Fanjul Peyró, 2008). Behar (2010) desvela la importancia de las televisoras, afirmando que en estudios donde se analizaban por su influencia durante un mes (139 varones y 82 mujeres ajenos al modelaje), se encontró que 69\% de las mujeres entraban en la clasificación de "muy delgado", lo que sólo sucedía en el 17.5\% de los hombres que se presentaban en la pantalla. En cuanto a sujetos obesos 
televisivos, los varones constituían el $25.5 \%$ y las mujeres el 5\%; por lo tanto, el modelo de moda suministrado por la televisión es el de la mujer sumamente delgada y joven. Crispo, Figueroa y Guelar (1998) mostraron datos entre los años de 1986 y 1996 sobre la cantidad de artículos sobre dietas hipocalóricas que aparecieron en las revistas de tirada masiva, los cuales aumentaron en un $70 \%$. Todos estos datos fueron pertenecientes al milenio anterior. En una comparación con datos de la actualidad, Jiménez-Cruz (2010) trabajó con mujeres quienes fueron expuestas a imágenes de mujeres modelos delgadas, las cuales manifestaron emociones más negativas hacia sus cuerpos que aquellas que observaron imágenes de mujeres con sobrepeso.

Los hombres, por su parte, han comenzado a creer que al desarrollar con vehemencia una gran masa muscular pueden resultar atractivos para las mujeres (Camacho Ruiz, Escoto Ponce de León, Contreras Landgrave, Ibarra Espinosa, \& García Rodríguez, 2012). Estos mismos autores realizaron un estudio el cual reveló que, entre fisicoculturistas, el $74.3 \%$ de usuarios presenta insatisfacción respecto a su imagen corporal y el $18.4 \%$ una motivación por incrementar su musculatura. Finalmente, la publicidad e internet despliegan miles de anuncios, creando necesidades y vendiendo cuerpos delgados para después promocionar la venta de otros productos como alimentos dietéticos, medicamentos que ayudan a adelgazar, así como técnicas de modelado del cuerpo tales como cirugías y lipoaspiración (Bazán \& Miño, 2015).

\section{Hábitos Alimenticios}

Un hábito alimenticio se denomina como la combinación de los alimentos y las bebidas que constituyen la ingesta dietética completa de una persona en el transcurso del tiempo (U.S. Department of Agriculture \& U.S. Department of Health and Human Services, 2010). Cruz-Sáez, Salaberria, Rodríguez y Echeburúa (2013) señalan que el intento de alcanzar un estereotipo de belleza esbelto conduce al seguimiento de dietas (en la mayoría de las ocasiones dietas "milagro", "De la luna" o cualquier tipo de dieta que no lleva el respaldo de una supervisión profesional), y en última instancia puede dar lugar a trastornos de conducta alimentaria (TCA). Por otro lado, la autoestima se ve afectada en ocasiones debido al estricto régimen de alimentación, lo que en momentos puede llevar a la aparición de pensamientos negativos (tristeza, cólera, auto-desprecio) una vez que éstas llegan a romperse (HidalgoRasmussen, Martín, \& Aguilera Cervantes, 2012). En una nota realizada por el diario Capital, Mayen (2018) detalla que en México, en los últimos 20 años, la anorexia se ha disparado un 300\% en los jóvenes, señalando 20 mil casos de anorexia cada año en la población nacional mexicana. Estos factores pudiesen ser más marcados en los modelos profesionales que se encuentran inmersos en un mundo donde su imagen, hábito alimenticio y autoestima lo es todo, e incluso su propia imagen puede usarse para servir de ejemplo al resto de la sociedad. En el siglo pasado algunos estudios fueron realizados en modelos, por ejemplo, un estudio dónde analizaron 
a un grupo de 18 'top models' en los años 90, en el cual dos presentaron bajo peso, 15 de ellas tenían un peso compatible con la anorexia y solo una presentaba un peso normal (Valles, 2013). Aunque se ha hallado una correlación significativa entre los patrones (hábitos) de alimentación en personas dedicadas al modelaje de moda no existe dato alguno en la actualidad riguroso y metodológico que respalde sistemáticamente la afirmación (Bogár \& Túry, 2018). Pero, se puede afirmar que las múltiples observaciones longitudinales de profesionales, dietas para perder peso, así como la inclusión en programas de ejercicio físico, la alimentación, el ideal de delgadez y la influencia de los medios de comunicación pueden influenciar en los desórdenes alimenticios y la disminución de peso corporal en las modelos profesionales (Rodgers, Ziff, Lowy, Yu, \& Austin, 2017). Se han dado ha conocer múltiples estudios de caso donde modelos de moda relatan sus restricciones alimenticias (Silva et al., 2013). Un aproximado en ésta restricción alimenticia en una modelo es de un $52 \%$ de ingesta calórica total. Con el apoyo del software de análisis de alimentos, aunque no es una mala alimentación, si se puede considerar en el límite de baja para una persona de 19 años o mayor que necesita hasta un $65 \%$ de ingesta calórica total (FoodChoices, 2009), lo cual se determina como una deficiencia en los hábitos alimenticios (Melin, Torstveit, Burke, Marks, \& Sundgot-Borgen, 2014). En el género masculino, los hábitos alimenticios no son de restricción, sino que conllevan a tener un aumento de ingesta alimenticia, como alto consumo de proteínas y la utilización de suplementos o esteroides con la finalidad de lograr un cuerpo inalcanzable (Mazón, Atiencia, \& Cabrera, 2017), además de la búsqueda de reducción de grasas en alimentos (Goldfield et al., 2010). Retomando el estudio realizado por San Mauro et al. (2014), se revela que de los 156 hombres de su muestra, el 56\% se fijan en la información nutricional de sus alimentos, el 52\% hizo mención de tener interés en lecturas sobre temas de nutrición y el $28 \%$ reportó el consumo con mucha frecuencia de suplementos alimenticios para aumentar la masa muscular.

El objetivo de la presente investigación se centra en analizar si existe relación de los medios de comunicación y hábitos alimenticios en los niveles de autoestima e imagen corporal. Al mismo tiempo, analizar si existe relación entre los medios y hábitos de alimentación, estado anímico e imagen de corporalidad; con éste propósito, se buscó encontrar diferencias significativas de las características antes descritas en hombres y mujeres, también diferencias significativas en tiempo dedicado al modelaje de moda y edad.

\section{Método}

\section{Diseño}

En la presente investigación, se utilizó el diseño de una investigación no experimental-transversal, del tipo correlacional-cuantitativo. 


\section{Participantes}

Se encontró una muestra probabilística de 100 participantes, 50 hombres y 50 mujeres $(\mathrm{N}=100)$ provenientes de institutos de modelaje de moda en la Ciudad de México, que han laborado en el modelaje de moda en al menos los últimos 6 meses a la realización de éste estudio.

\section{Instrumentos}

Para llevar a cabo la evaluación multidimensional se contó con el apoyo de cinco instrumentos para analizar las variables mencionadas. Dichos instrumentos son: el "Cuestionario de Influencias sobre el Modelo Estético Corporal” (CIMEC), usando versión para hombres (Vázquez et al., 2010), y para mujeres (Vázquez, Álvarez, \& Mancilla, 2000). La versión femenina cuenta con un coeficiente Alfa de Cronbach de .94, mientras que la versión masculina posee las propiedades internas de .90 en el Coeficiente de Alfa de Cronbach. Su uso fue para la medición de las variables de influencia de medios de comunicación. El test para autoestima de Rosenberg (1965), que consta de 10 reactivos, cuenta con un coeficiente Alfa de Cronbach de .89 y un coeficiente de correlación test-retest a dos semanas de .91 para la medición del estado anímico. El Test de Conductas Alimenticias (Garner \& Garfinkel, 1979) "EAT" por sus siglas en inglés para el análisis de hábitos alimenticios y sus restricciones de ingestas alimenticias, instrumento que cuenta con una confiabilidad de Alfa de Cronbach de .87, para la medición de anorexia y bulimia en mujeres. El Inventario de Dismorfia Muscular (Rhea, Lantz, \& Cornelius, 2004), "MDI" por sus siglas en inglés, el cual está basado en una escala tipo Likert con 27 reactivos y con seis opciones de respuesta. Se usó éste instrumento por su apartado de conducta alimenticia en varones. Cuenta con una confiabilidad de .72 en el Alfa de Cronbach. El test MBSQR (Botella García del Cid, Ribas Rabert, \& Ruiz, 2009), versión española, adaptada para la variable de imagen corporal: éste instrumento cuenta con las propiedades de confiabilidad de .88 en el Alfa de Cronbach y un coeficiente de correlación test-retest de .77.

\section{Procedimiento}

Siguiendo las normas éticas a lo largo de toda la investigación, se estableció contacto con los directores de la agencia de modelaje, para obtener el acceso a los participantes gracias a los dirigentes. Se llevó a cabo el informe sobre el tema de la investigación así como los objetivos de ésta respecto a los temas de interés. Una vez notificados sobre el estudio y obtenido el consentimiento informado, accedieron los modelos de moda a contestar los instrumentos de medición. Al mismo tiempo se detalló la creación de bases de datos para el análisis de resultados, recalcando la confidencialidad de sus datos y la imposibilidad de revelación acerca de su información personal o el nombre de la agencia que otorgó el acceso. La aplicación 
de los instrumentos (CIMEC, EAT, MDI, MBSQR y Rosenberg) fue de manera individual, aclarando las instrucciones y dudas. Los rangos en tiempos de ejecución de las pruebas fueron de 30 a 45 minutos. Posteriormente, se pasó al registro y codificación de datos.

\section{Análisis Estadísticos}

Para el análisis de los resultados se utilizó el paquete estadístico SPSS v.20.0. Se analizaron las cinco pruebas psicológicas en las cuales se muestran las diferencias significativas entre hombres y mujeres, así como las diferencias de nivel, las correlaciones de la imagen corporal con autoestima, imagen corporal con hábitos alimenticios, imagen corporal con medios de comunicación, así como los datos percibidos en relación de autoestima y las variables de medios de comunicación y hábito de alimentación. Por último, se analizó la edad de los participantes en relación a las variables ya descritas.

\section{Resultados}

La edad de los participantes oscila entre los 18 años de edad como mínimo y 27 años como máximo en ambos géneros, con una media de edad de 21 años. Tomando como base los años que los modelos han dedicado a su trabajo (Tabla 1) se contempla un tiempo considerable para el estudio, ya que muchos poseen la estancia para poder analizar los factores a estudiar durante esa trayectoria. El resultado es un promedio general de 2.64 años de la muestra analizada.

Tabla 1

Descripción de tiempo dedicado al mundo de modelaje

\begin{tabular}{lccc}
\hline Años en labor de modelaje $(\mathrm{N}=100)$ & Mínima & Máxima & Media \\
Años de modelaje en muestra general & 1 & 7 & 2.64 \\
Hombres & 1 & 7 & 2.48 \\
Mujeres & 1 & 5 & 2.80 \\
\hline
\end{tabular}

En la Tabla 2.1 se muestra que ambos géneros poseen un grado considerable de afección en el estado anímico. Aunado a esto, la influencia de medios de comunicación revela que el género femenino se ve más afectado por los constantes anuncios que se dan día a día. En el sector de hábito alimenticio, se muestra que los hombres poseen un régimen alimenticio más estricto. Sin embargo, se debe considerar que no es restricción alimenticia, sino que se trata de uso de suplementos y alimentos que aumenten su corporalidad. Debido a que en el caso de 
las mujeres se habla de una restricción, al hablar de imagen corporal se observa como casi emulada la cantidad de preocupación de la imagen en ambos géneros, dando como resultado una cantidad mayor en el caso masculino; sin embargo, dicha cantidad no es de gran diferencia.

Tabla 2.1

Descripción de niveles de autoestima, imagen corporal, medios de comunicación y hábitos alimenticios

\begin{tabular}{|c|c|c|c|c|}
\hline \multirow{5}{*}{ Autoestima } & \multirow[b]{3}{*}{ Elevada } & \multicolumn{2}{|c|}{ Modelos } & \multirow{2}{*}{ Total } \\
\hline & & Fem. & Masc. & \\
\hline & & 0 & 0 & 0 \\
\hline & Media & 11 & 11 & 22 \\
\hline & Baja & 39 & 39 & 78 \\
\hline Influencia de medios de & Presencia & 45 & 36 & 81 \\
\hline comunicación & Ausencia & 5 & 14 & 19 \\
\hline & Ordinario & 18 & & 18 \\
\hline Hábitos alimenticios femeninos & Alto riesgo & 32 & & 32 \\
\hline & Caso Clínico & 0 & & 0 \\
\hline Hábitos alimenticios & Ordinario & & 4 & 4 \\
\hline masculinos & Régimen alimenticio de riesgo & & 46 & 46 \\
\hline \multirow{3}{*}{ Imagen Corporal } & $\begin{array}{l}\text { Percepción buena de imagen } \\
\text { corporal }\end{array}$ & 5 & 4 & 9 \\
\hline & $\begin{array}{l}\text { Percepción media de imagen } \\
\text { corporal }\end{array}$ & 3 & 2 & 5 \\
\hline & $\begin{array}{l}\text { Percepción mala de imagen } \\
\text { corporal }\end{array}$ & 42 & 44 & 86 \\
\hline
\end{tabular}

Las Tablas 2.2 y 2.3 presentan la información antes mencionada, donde se encontró que entre ambos géneros no existen diferencias significativas en imagen corporal y autoestima. 
Tabla 2.2

Análisis comparativo de imagen corporal por sexo

\begin{tabular}{lllllll}
\hline & Alta & Media & Baja & $t$ & gl & P \\
Mujeres & 31 & 15 & 14 & & & \\
Hombres & 25 & 19 & 16 & .179 & 98 & .858 \\
P: Valor de Alpha no significativo, gl: Grados de & \\
libertad, t: Valor de t independiente
\end{tabular}

Tabla 2.3

Análisis comparativo de autoestima por sexo

\begin{tabular}{llllccc}
\hline & Alta & Media & Baja & $\mathrm{t}$ & $\mathrm{gl}$ & $\mathrm{P}$ \\
Mujeres & 31 & 15 & 14 & & & \\
Hombres & 25 & 19 & 16 & 1.03 & 98 & .306 \\
\hline
\end{tabular}

P: Valor de Alpha no significativo, gl: Grados de libertad, t: Valor de t independiente

Las Tablas 3.1 y 3.2 muestran el análisis de correlación entre las variables. Cabe mencionar que se desarrollaron dos matrices: una para el género masculino y otra para el femenino. Esto, dado que los instrumentos utilizados poseían diferentes reactivos y algunos de ellos con mediciones destinadas a un cálculo diferente como lo es el hábito alimenticio. Los estudios permitieron encontrar muchas correlaciones significativas entre las variables. Encontramos correlaciones significativas negativas ( $\mathrm{r}$ de Pearson) entre la autoestima e imagen corporal en ambos géneros, los hábitos alimenticios con el estado anímico e imagen corporal en ambos géneros, medios de comunicación sobre las variables dependientes del lado femenino. También se vislumbra la relación que ejerce la misma imagen corporal sobre el estado anímico en general. No se encontraron correlaciones significativas entre el estado anímico y medios de comunicación de lado masculino pero se muestra una relación significativa en el género femenino entre el estilo alimenticio y medio de comunicación. 


\section{Tabla 3.1}

Correlación de variables en género masculino

\begin{tabular}{lcccc}
\hline \multicolumn{2}{l}{$\begin{array}{l}\text { Matriz de correlación de Pearson de las variables } \\
\text { comunicación, Hábitos alimenticios sobre }\end{array}$} & \multicolumn{3}{c}{ Imagen Corporal y Autoestima } \\
\hline & Medios de & Hábitos & Imagen & Autoestima \\
& comunicación & alimenticios & corporal & \\
Medios de & .132 & -.309 & .160 \\
comunicación & .360 & $.029^{\star}$ & .218 \\
Hábitos & & -.313 & -.334 \\
alimenticios & & $.027^{\star}$ & $.018^{*}$ \\
Imagen corporal & & & -.302 \\
& & & $.033^{*}$
\end{tabular}

Correlación de Pearson: $\mathrm{P}<.05^{*}$

Tabla 3.2

Correlación de variables en género femenino

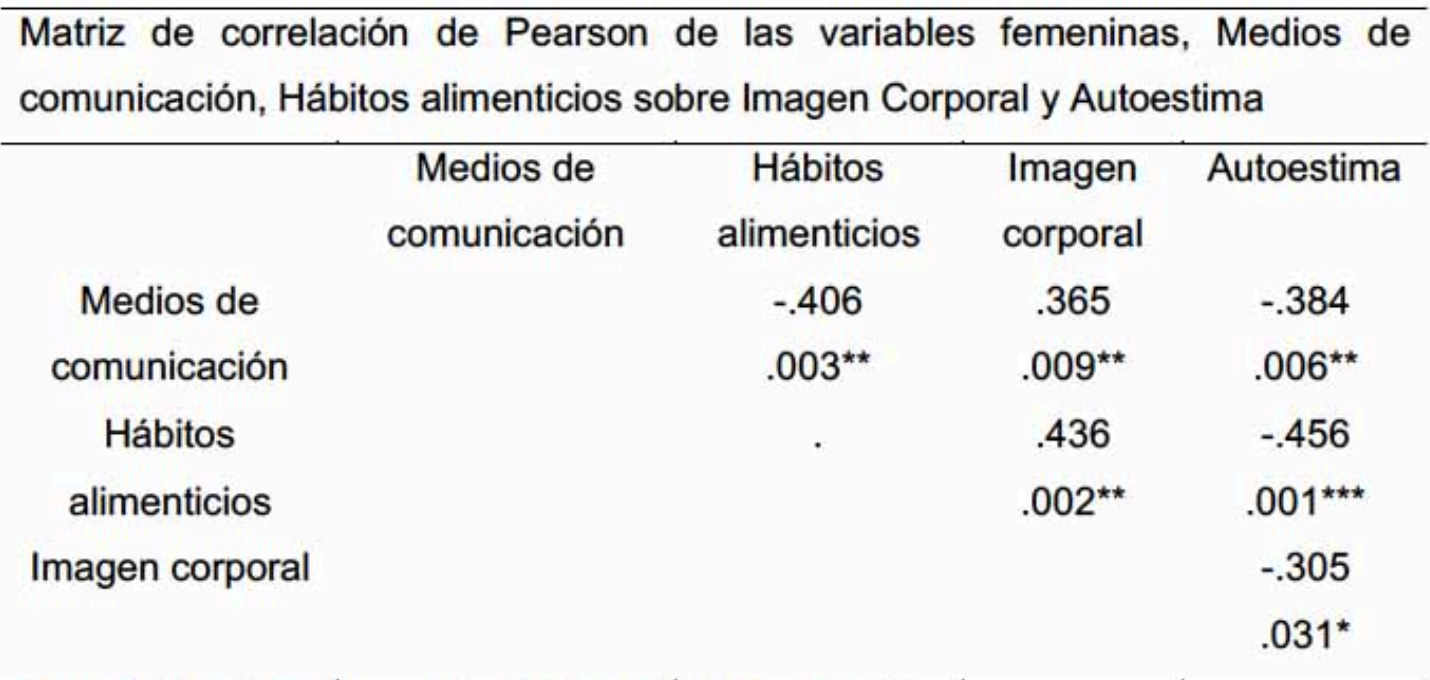

Correlación de Pearson: $\mathrm{P}<.05^{\star}, \mathrm{P}<.01^{\star \star}, \mathrm{P}<.001^{\star \star \star}$

No se halló una correlación significativa ni entre la edad o en el tiempo en el modelaje de los participantes con las cuatro variables analizadas.

\section{Discusión}

Esta investigación confirma que los modelos presentan una alteración en su estado anímico e ideas distorsionadas en cuanto a la imagen corporal. Estos, se ven influenciados por los medios de comunicación y 
hábitos alimenticios. Se estima que el $90 \%$ de las mujeres que ejercen la práctica del modelaje, serán susceptibles al dominio de los medios de comunicación e intentarán alcanzar dichos cánones de belleza. El porcentaje de modelos afectados para el género masculino desciende a $72 \%$; sin embargo, esos 7 de cada 10 en números globales, también pueden padecer esta influencia. Respecto al estilo alimenticio, el 64\% de las mujeres pueden llegar a padecer un trastorno alimentario de alto riesgo, tal como menciona Chacón Araya (2017), quien reportó un mayor control y menor ingesta de alimentos. El 92\% de los hombres también pueden padecer un trastorno alimenticio de riesgo, esta vez en alto consumo de alimentos y suplementos que los ayuden a alcanzar ese estándar corporal (Gómez, 2013). Respecto a la imagen corporal, el 84\% de las mujeres y el $92 \%$ de los varones pueden llegar a verse alterados de manera negativa debido a la alta influencia de estas variables. La autoestima del 78\% de ambos géneros (sin embargo esta cifra puede variar) puede verse reducida por el gran poder de los medios de comunicación. También su imagen corporal se ve afectada y pueden padecer considerables niveles de tristeza e incluso, en casos extremos, depresión severa (Bogár \& Túry, 2018).

\section{Limitaciones}

Dentro de las limitaciones de este trabajo está que la muestra es pequeña. Para seguir ampliando y detallando resultados significativos, debe considerarse el uso de muestras aún mayores de modelos. Por otro lado se reconoce como limitación que los instrumentos se aplicaron a la hora que los modelos estaban estresados y agotados.

\section{Conclusiones}

Sin duda caminar en una pasarela, ser objeto de fotografías publicadas en revistas, pareciera recalcar la belleza de una persona. Sin embargo, ese camino parece tener grandes riesgos. Debido al impacto anímico y al ideal distorsionado de corporalidad que las modelos proyectan, se requiere la intervención de especialistas, psiquiatras, nutriólogos y psicólogos para controlar y prevenir estos fenómenos. Este estudio puede servir como apoyo a futuras intervenciones, individuales o grupales que ayuden a los profesionales del modelaje. Por último la investigación en este campo debe ampliarse y difundirse para la prevención: sería importante que los niños, adolescentes y adultos, conozcan los riesgos que puede tener la carrera de un modelo de moda profesional.

\section{Referencias}

Bazán, C. I., \& Miño, R. (2015). La imagen corporal en los medios de comunicación masiva. Psicodebate. Psicología, Cultura y Sociedad, 15(1), $23-42$. 
Behar, R. (2010). La construcción cultural del cuerpo: El paradigma de los trastornos de la conducta alimentaria. Revista Chilena de NeuroPsiquiatria, 48(4), 319-334. http://dx.doi.org/10.4067/S0717-9227201 0000500007

Bogár, N., \& Túry, F. (2018). Abusing the body: Psychological abuse? The bioethical aspects of the fashion model profession. Journal of Obesity \& Eating Disorders, 4(1), 1-4. https://doi.org/10.21767/2471-8203.10003 5

Botella García del Cid, L., Ribas Rabert, E., \& Ruiz, J. B. (2009). Evaluación psicométrica de la imagen corporal: Validación de la versión española del multidimensional body self relations questionnaire (MBSRQ). Revista Argentina de Clínica Psicológica, 8(3), 253-264.

Camacho Ruiz, E. J., Escoto Ponce de León, M. D. C., Contreras Landgrave, G., Ibarra Espinosa, M. L., \& García Rodríguez, J. (2017). Relación entre motivación por la musculatura e indicadores antropométricos en fisicoconstructivistas. Revista Mexicana de Investigación en Psicología, 4(S1), 112-119.

Chacón Araya, Y. (2017). Descripción de la figura fisica y composición corporal, imagen corporal, hábitos de alimentación, de actividad física y opinión acerca de cirugias cosméticas en modelos costarricenses (Tesis doctoral). Universidad de Olavide, España.

Crispo, R., Figueroa, E., \& Guelar, D. (1998). Anorexia y bulimia. Lo que hay que saber. Barcelona, España: Gedisa.

Cruz-Sáez, M. S., Salaberria, K., Rodríguez, S., \& Echeburúa, E. (2013). Imagen corporal y realización de dieta: Diferencias entre adolescentes españolas y latinoamericanas. Universitas Psychologica, 12(3), 699-708.

Díaz Soloaga, P., Quintas Froufe, N., \& Muñiz, C. (2010). Cuerpos mediáticos versus cuerpos reales. Un estudio de la representación del cuerpo femenino en la publicidad de marcas de moda en España. ICONO 14, Revista de Comunicación y Tecnologias Emergentes, 8(3), 244-256.

Esteves, M. (2012). Relación entre la insatisfacción con la imagen corporal, autoestima, auto concepto fisico y la composición corporal en el alumnado en el segundo ciclo de educación secundaria de la ciudad de Aliacante (Tesis doctoral). Granada, España.

Fanjul Peyró, C. (2008). Modelos masculinos predominantes en le mensaje publicitario y su influencia social en la psicopatología del siglo XXI: La vigorexia. Prisma Social, (1), 1-26.

FoodChoices (Version 6.00.2258) [Software de computación] (2009). Brisbane, Australia: Xyris Software.

Garner, D. M., \& Garfinkel, P.E. (1979). The Eating Attitudes Test: An index of the symptoms of anorexia nervosa. Psychological Medicine, 9(2), 273-279. https://doi.org/10.1017/S0033291700030762

Garner, D. M., Garfinkel, P. E., Schwartz, D., \& Thompson, M. (1980). Cultural expectations of thinness in women. Psychological Reports, 47(2), 483-491. https://doi.org/10.2466/pr0.1980.47.2.483

Gil Madrona, P. (2013). La satisfacción corporal y su rol en las relaciones sociales. Universitas Psychologica, 12(2), 547-558.

Goldfield, G. S., Moore, C., Henderson, K., Buchholdz, A., Obeid, N., \& Flament, M. F. (2010). Body dissatisfaction, dietary restraint, depression, 
and weight status in adolescents. Journal of School Health, 80(4), 186-192. https://doi.org/10.1111/j.1746-1561.2009.00485.x

Gómez, G. (2013). Imagen corporal y orientación sexual: Deseo de un modelo corporal masculino ideal. CDMX, México: Fénix.

Hidalgo-Rasmussen, C. A., Martín, H. S., \& Aguilera Cervantes, V. G. (2017). Percepción del peso corporal, comportamientos alimentarios y calidad de vida en estudiantes mexicanos. Revista Mexicana de Investigación en Psicología, 4(S1), 80-90.

Jiménez-Cruz, B. E. (2010). Riesgo para trastorno alimentario, ansiedad, depresión y otras emociones asociadas a la exposición de imágenes publicitarias. Anales de Psicologial Annals of Psychology, 26(1), 11-17.

Leit, R. A., Gray, J. J., \& Pope, H. G. (2002). The media's representation of the ideal male body: A cause for muscle dysmorphia?. International Journal of Eating Disorders, 31(3), 334-338. https://doi.org/10.1002/eat.10019

Mayen, V. (16 de abril de 2018). Las cifras de anorexia y bulimia en México. Capital, p. 1. Recuperado de https://www.capitalmexico.com.mx/socieda $\mathrm{d} /$ cifras-anorexia-bulimia-mexico-suicidio-jovenes-salud-tratamiento/

Mazón, J. P., Atiencia, L. M., \& Cabrera, H. (2017). Factores psicológicos asociados a la vigorexia en los usuarios de gimnasios del Azuay y en el periodo 2016-2017. Revista Electrónica de Psicología Iztacala, 20(3), 131-134.

Melin, A., Torstveit, M. K., Burke, L., Marks, S., \& Sundgot-Borgen, J. (2014). Disordered eating and eating disorders in aquatic sports. International Journal of Sport Nutrition and Exercise Metabolism, 24(4), 450-459. http s://doi.org/10.1123/ijsnem.2014-0029

Meyer, B., Enström, M. K., Harstveit, M., Bowles, D. P., \& Beevers, C. G. (2007). Happiness and despair on the catwalk: Need satisfaction, wellbeing, and personality adjustment among fashion models. The Journal of Positive Psychology, 2(1), 2-17. https://doi.org/10.1080/1743976060107 6635

Nieves, I. C. R. (2009). La Imagen corporal: Hacia una construcción social para la psicología industrial organizacional. Poiésis, 9(18), 1-6.

Padrón-Salas, A., Hernández-Gómez, J. F., Nieto-Caraveo, A., Aradillas-García, C., Cossío-Torres, P. E., \& Palos-Lucio, A. G. (2015). Asociación entre autoestima y percepción de la imagen corporal en estudiantes universitarios. Acta Universitaria, 25(1), 76-78.

Prieto Vela, A., \& Muñoz-Najar Pacheco, A. O. (2015). Bienestar subjetivo e imagen corporal en estudiantes universitarias de Arequipa. Liberabit, 21(2), 321-328.

Raich, R. M. (2004). Una perspectiva desde la psicología de la salud de la imagen corporal. Avances en Psicologia Latinoamericana, 22(1), 15-27.

Rhea, D. J., Lantz, C. D., \& Cornelius, A. E. (2004). Development of the muscle dysmorphia inventory (MDI). Journal of Sports Medicine and Physical Fitness, 44(4), 428-435.

Rodgers, R. F., Ziff, S., Lowy, A. S., Yu, K., \& Austin, S. B. (2017). Results of a strategic science study to inform policies targeting extreme thinness standards in the fashion industry. International Journal of Eating Disorders, 50(3), 284-292. https://doi.org/10.1002/eat.22682 
Rosenberg, M. (1965). La sociedady la imagen de simismo adolescente. Princeton, NJ, US: Princeton University Press.

San Mauro, I., Garicano, E., Gonzáles, M., Villacorta, P., Megias, A., Miralles, B., Figueroa, M., Andrés, N., Bonilla, M, A., Arranz, P., Bernal, M, D., Ruiz, A, M., Moraleda, E., \& De la Calle, L. (2014). Hábitos alimentarios y psicológicos en personas que realizan ejercicio físico. Nutrición Hospitalaria, 30(6), 1327-1329.

Saüch, G., \& Castañer, M. C. (2013). La proyección de la imagen corporal en jóvenes adultos y en la tercera edad. Una aplicación específica de expresión de la corporalidad. Retos: Nuevas Tendencias en Educación Física, Deporte y Recreación, (24), 113-116.

Silva, D. R., Ribeiro, A. S., Pavão, F. H., Ronque, E. R., Avelar, A., Silva, A. M., \& Cyrino, E. S. (2013). Validity of the methods to assess body fat in children and adolescents using multi-compartment models as the reference method: A systematic review. Revista da Associação Médica Brasileira, 59(5), 475-486. http://dx.doi.org/10.1016/j.ramb.2013.03.00 6

Soto Ruiz, M., Marín Fernández, B., Aguinaga Ontoso, I., Guillén-Grima, F., Serrano Monzó, I., Canga Armayor, N., ... \& Annan, J. (2015). Análisis de la percepción de la imagen corporal que tienen los estudiantes universitarios de Navarra. Nutrición Hospitalaria, 31(5), 2269-2275. http ://dx.doi.org/10.3305/nh.2015.31.5.7418

Swami, V., \& Szmigielska, E. (2013). Body image concerns in professional fashion models: Are they really an at-risk group? Psychiatry Research, 207(1-2), 113-117. https://doi.org/10.1016/j.psychres.2012.09.009

U.S. Department of Agriculture \& U.S. Department of Health and Human Services. (2010). Dietary Guidelines for Americans 2010. Washington, WA, US: Sky Pony Press.

Valles, M. (2013). La imagen corporal: Programa preventivo sobre los trastornos de conducta alimentaria (Tesis doctoral). Universidad de Castilla la Mancha, España.

Vázquez, R., Alvarez, G., \& Mancilla, J. M. (2000). Consistencia interna y estructura factorial del Cuestionario de Influencia de los Modelos Estéticos Corporales (CIMEC), en población mexicana. Salud Mental, 23(6), 18-24.

Vázquez, R., Velásquez, G., López, X., Álvarez, G. L., Mancilla, J. M., \& Franco, K. (2010). Consistencia interna y estructura factorial del cuestionario de Influencias del modelo estético corporal (CIMEC) en estudiantes mexicanos. Psicología y Ciencia Social, 12(1), 5-11.

Notas

[1] Universidad Tecnológica de México-Atizapán (UNITEC-ATIZAPÁN), Blvrd Calacoaya 7, La Ermita, 52999 Cd López Mateos, México. Correo electrónico: gilbertopen1993@gmail.com 